\title{
Robust Decisions of Capital-Constrained Supply Chain with Demand Uncertainty
}

\author{
Nina Yan
}

\begin{abstract}
In this paper, we design a supply chain finance system composed of a capital-constrained retailer, a manufacturer and a commercial bank, with unknown demand distribution. Considering the retailer's capital-constraint degree, credit line and bankruptcy risk, we establish a Stackelberg game in which the manufacturer acts as the leader and the commercial bank as the sub-leader. Based on the profit-maximization objective, we solve the optimal order of the retailer, the optimal interest rate of the commercial bank and the optimal wholesale price of the manufacturer and the robust deviation for each participant based on minimize-maximum-regret. Finally, we conduct numerical examples to make a comparative analysis between these two different decisions and explore the impact of the demand uncertainties on optimal strategies and robust deviations. It concluded that the robust decision provides a conservative strategy for risk aversion and the region of demand intervals has great impact on robust deviations, rather than the optimal decisions.
\end{abstract}

Index Terms-Supply chain finance, robust optimization, Stackelberg games, demand uncertainty.

\section{INTRODUCTION}

Supply chain financing (SCF) is a nascent area emerging from a growing appreciation for financial flows by supply chain practitioners and by researchers, which is also a response to an emerging need for banks to play a more active role in the activities of suppliers and buyers [1]. Well-structured SCF schemes have proven valuable and indispensable to accelerate the turnover of inventories and receivables to free up more cash.

In the past, academic papers regarding supply chain management (SCM) mainly dealt with the design and optimization of the flows of goods and information. The financial flows between companies in the supply chain, however, were often neglected and have only recently found greater attention in the academic SCM literature. Until recently, financial considerations were conspicuously scarce in the extensive literature on models of supply chain operations.

The research in the SCF field refers to multi-disciplines, such as financial engineering, corporate finance, logistics management, SCM and operation management. Dada and $\mathrm{Hu}$ (2008) also analyzed the decision of a capital-constrained

Manuscript received January 31, 2014; received May 5, 2014. This work was supported in part by the Natural Science Foundation of China (Grant No. 71002021, 71372191), Beijing Philosophy and Social Science Planning Program (grant no.12JGC099) and program for Innovation Research in Central University of Finance and Economics.

Nina Yan is with the Central University of Finance and Economics, Beijing, 100081, P.R. China (e-mail: yannina@ cufe.edu.cn). newsvendor who can borrow funds for procurement; from this study, we derive a non-linear loan schedule that coordinates the channel [2]. Chen and Wang (2012) investigate the impacts of limited liability for the performance of SCF and shows that limited liability accounts for the reason why the retailer with a lower initial budget initiates a higher ordering level under trade credit contract [3]. Jing et al., (2012) discuss the equilibrium in SCF with two credit choices (bank or trade credit) and show that bank credit financing generally charges a lower wholesale price and thus becomes more attractive than trade credit financing for the retailer [4]. Zhang (2011) considered a multi-product newsboy problem with both supplier quantity discounts and a budget constraint. The problem is formulated as a mixed integer nonlinear programming model and solved using a Lagrangian relaxation approach [5]. Lee and Rhee (2010) focused on the coordination problems of capital-constrained supply chain agents with positive inventory financing costs and investigated four widely examined coordination mechanisms [6]. Srinivasa and Mishra (2011) considered a two-level supply chain with a single retailer and manufacturer, where both firms are facing financial constraints. They investigated how the lender's profit related to the cash position at the next level of the supply chain of the borrowers [7].

However, our world is increasingly uncertain and vulnerable. In the SCF system, the uncertainties of the inner operations of supply, production and sales and outer demand fluctuations would have inevitable effects on both operational decisions and financial decisions. With the increases of demand uncertainties, most companies would exert themselves to implement some SCF strategies with robust performance for mitigating emergency risks. Although a large number of researchers have engaged on using robust optimization method to solve newsvendor problems, resource allocation problems, inventory problems, pricing decision problems, little research focus on the robust decisions of capital-constrained supply chain in which the operational decisions and financial decisions are integrated together.

In this paper, we mainly focus on three issues. First, we design an SCF system composed of a financially constrained retailer, a manufacturer and a commercial bank as the "capital supplier". In this system framework, we formulate inter-functional and inter-organizational activities as multi-level Stackelberg games in which the manufacturer acts as the leader and the bank as the sub-leader. We characterize the equilibrium solution for each partner and present comparative statics both analytically and numerically. Second, from the point of view of demand uncertainty, we discuss the robust decisions of SCF system based on 
minimize-maximum-regret, only knowing the region of demand intervals. Third, to compare with the decisions of optimal objective and robust deviation, we conduct numerical analysis to explore the impact of demand uncertainty on SCF decisions and make a comparative analysis between them.

The rest of the paper is organized as follows. Section II presents the basic modeling of SCF, including the framework, notations and assumptions. Section III formulates a Stackelberg game of SCF and analyzes optimal decisions for each participant. In Section IV, we analyze the deviation robustness of SCF decisions. Section V conducts numerical studies to illustrate our analytical results. Finally, Section VI concludes with remarks.

\section{MODEL DESCRIPTION}

In this paper, we set up a stylized single-period newsvendor model of the SCF system involving three parties: a capital-constrained retailer, an upstream manufacturer, and a commercial bank. In our model, the retailer faces a situation equivalent to the classical newsvendor, where he purchases a single product from the manufacturer and then sells it to his customers, not knowing the actual demand for the product at the time of purchase. The most common and practical problem is that the retailer is capital-constrained in the procurement process. To ease financial needs and help settle payments on time he chooses SCF as a means of substituting for lower creditworthiness.

To clearly describe and analyze our quantitative models, we first summarize the notations and assumptions used below.

$q$ is order quantity, retailer's decision variable. $w$ is the unit wholesale price, manufacturer's decision variable. $R_{r}$ is bank's exogenous interest rate. The exogenous parameters are the risk free rate $R_{f}$, the unit retailer price $p$, the unit production cost $c$ and the retailer's initial capital $K_{r} . \pi(\cdot)$ is the expected profit for each decision-maker. In addition, we use superscripts $N$ and $B$ for no financing and bank financing, and superscripts "*" and $R$ for optimal decision and robust decision, respectively. Moreover, the subscript $r, m$ and $b$ are used for the retailer, manufacturer and the bank, respectively.

In our model, we assume the product is perishable and has zero salvage value by the end of the period. To simplify exposition, we also ignore any goodwill loss due to stockout. Moreover, without loss of generality, we assume $c \leq w$ and $w\left(1+R_{r}\right) \leq p$ are justified.

Following the convention in the bank credit literature, we assume the bank market is competitive and that the risk-neutral banks have access to unlimited funds at the risk-free interest rate $R_{f}$, which is normalized to zero without loss of generality[4], [8], [9]. We also consider the perfect capital market without taxes, transaction costs and bankruptcy costs.

Furthermore, the uncertain demand is denoted by a non-negative random variable $x$. The probability density function (PDF) is $f(\cdot)$, cumulative distribution function
(CDF) is $F(\cdot)$, and complementary $\mathrm{CDF}$ is $\bar{F}(\cdot)=1-F(\cdot)$. Let $F$ be differentiable, strictly increasing and $F(0)=0$. For technical purpose, we assume that the demand distribution has a strictly increasing failure rate (IFR), i.e. $G(x) \equiv x f(x) / \bar{F}(x)$ is monotonically increasing in $x$, which is a common assumption in supply chain modeling. Many common distributions are IFR, for instance, the uniform, normal, exponential, gamma and so on (see to Lariviere, 2006). Moreover, without loss of generality, we assume that $p \geq w\left(1+R_{r}\right) \geq c\left(1+R_{r}\right)$ is justified.

In addition, we also assume that both the manufacturer and the retailer are assumed to be risk- neutral with the objective of maximizing their expected profits, and all the participants have the same belief about the distribution of demand, namely, the information on demand is symmetric.

\section{Optimal Decisions of SCF}

Considering the power of channel allocation and mastery of resources, we formulate the interaction between the capital-constrained retailer, the manufacturer and the bank as a multi-level Stackelberg game in which the manufacturer acts as the leader and the bank acts as the sub-leader.

The sequence of moves is as same as follows. Firstly the leader (manufacturer) will announce how much charge the retailer for the wholesale price $w$ and then the sub-leader (bank) will accordingly evaluate the retailer's financing conditions (e.g., initial capitals, bankruptcy risks, procurement/production quantities and so on) and set a suitable interest rate $R_{r}$ to the retailer. Then, act as the follower, the retailer will decides how much to order according to the bank's interest rate and the manufacturer's wholesale price. To pursue the Stackelberg equilibrium results, we use backward induction to solve the retailer's optimal decision first.

\section{A. Retailer's Optimal Decision}

We use $q^{B}$ to denote the order quantity with bank financing and the loan amount from the bank is $L\left(q^{B}\right)=w q^{B}-K_{r}$. Hence, the capital-constrained retailer's problem with financing can be formulated as Eq. (1).

$$
\begin{gathered}
\max _{w q^{B} \leq K_{r}} \pi_{r}^{B}=E\left[p \min \left(q^{B}, x\right)-L\left(1+R_{r}\right)\right]^{+}-K_{r} \\
\text { s.t. } w q^{B} \leq K_{r}
\end{gathered}
$$

From Equation (1), it's obvious that If the retailer's liquid assets from the sales at the end of the season can cover its loan obligations, i.e., $E\left[p \min \left(q^{B}, x\right)\right] \leq L\left(q^{B}\right)\left(1+R_{r}\right)$, he will encounter bankruptcy risk. So from this expression we can easily get the minimal realized demand $\hat{x}\left(q^{B}\right)=\left(w q^{B}-K_{r}\right)\left(1+R_{r}\right) / p=L / \hat{p}$ and the maximal order quantity $\hat{q}=K_{r} / w$, where $\hat{p}=p / 1+R_{r}$ is the unit discounted revenue with financing. Obviously, when the realized demand of customers is too low or the order quantity 
is too high the retailer would take a large backlog of inventory, which may result in low revenues. In this case, the retailer couldn't repay the loans and would face the risk of bankruptcy. To some extent, the bankruptcy risk would transfer to the bank.

Proposition 1. For increasing failure rate (IFR) distributions of demand, when the bank sets $R_{r}$ and the manufacturer sets $w$, the retailer's optimal order quantity $q^{B^{*}}$ after financing satisfies that $\hat{p} \bar{F}\left(q_{r}^{B^{*}}\right)=w \bar{F}\left(\hat{x}\left(q_{r}^{B^{*}}\right)\right)$ when $K_{r} \leq w \bar{F}^{-1}(w / \hat{p})$.

Proof. Eq. (1) Can be rewritten as follows, $\pi_{r}^{B}=-K_{r}-L\left(1+R_{r}\right) \bar{F}\left(\frac{L\left(1+R_{r}\right)}{p}\right)+p \int_{\hat{x}\left(q^{B}\right)}^{q^{B}} x f(x) d x+p q^{B} \bar{F}\left(q^{B}\right)$ $=p \int_{\hat{x}\left(q^{B}\right)}^{q^{B}} \bar{F}(x) d x-K_{r}$.

If the distribution of demand is IFR, if $K_{r} \leq w \bar{F}^{-1}(w / \hat{p})$, taking the first-order and second-order derivative of $\pi_{r}^{B}$ with respect to $q^{B}$, it follows that $d \pi_{r}^{B} / d q^{B}=p\left[\bar{F}\left(q^{B}\right)-w / \hat{p} \bar{F}(\hat{x})\right]$ and $d^{2} \pi_{r}^{B} / d q^{B 2}=p\left[-f\left(q^{B}\right)+(w / \hat{p})^{2} f(\hat{x})\right]<0$. Therefore, from $d \pi_{r}^{B} / d q^{B^{*}}=0$ we have $q^{B^{*}}=\bar{F}^{-1}[w \bar{F}(\hat{x}) / \hat{p}]$.

It follows from Proposition 1 that the capital-constrained retailer will not borrow from the bank when $\hat{p}<w<\bar{F}\left(K_{r} / w\right)$. In order to encourage the retailer to adopt bank financing, the bank chooses $\hat{p}$ between $w / \bar{F}\left(K_{r} / w\right) \leq \hat{p}<p$ where $\hat{p}<p$ is equivalent to $R_{r}>0$.

\section{B. Bank's Optimal Decision}

In this paper, we assume that the bank has abundant capital to satisfy the retailer's loan request. Furthermore, based on the marketization of interest rate, we formulate the bank's decision as a profit-maximization problem by choosing an optimal interest rate.

$$
\max _{R_{r}} \pi_{b}\left(R_{r} ; q^{\beta^{*}}, x\right)=\min \left\{L\left(q^{\beta^{*}}\right)\left(1+R_{r}\right), p \min \left(q^{\beta^{*}}, x\right)\right\}-L\left(q^{\beta^{*}}\right)\left(1+R_{f}\right)
$$

Note, at the end of the selling season if the retailer is faced with a bankruptcy risk, i.e., the revenue is insufficient to cover the loan; the bank would undertake the bankruptcy risk and has the priority of liquidation preferences to acquire all the sales revenue of the retailer.

Proposition 2. If the demand distribution is IFR, considering the retailer's initial capital and bankruptcy probability, then the commercial bank will set an optimal interest rate $R_{r}^{*}$ can be expressed as follows:

$$
R_{r}^{*}=w^{2}\left(p-\operatorname{Lg}\left(\hat{x}\left(q^{B^{*}}\right)\right)\right)-p^{2}\left(w \bar{F}\left(q^{B^{*}}\right)-\operatorname{Lf}\left(q^{B^{*}}\right)\right) / w^{2} \operatorname{Lg}\left(\hat{x}\left(q^{B^{*}}\right)\right) .
$$

Proof. Eq. (2) can be rewritten as $\pi_{b}=p \int_{0}^{\hat{x}\left(q^{B^{*}}\right)} \bar{F}(x) d x-w q^{B^{*}}+K_{r}=p \int_{0}^{\hat{x}\left(q^{B^{*}}\right)} \bar{F}(x) d x-\hat{p} \hat{x}\left(q^{B^{*}}\right)$. Taking the first-order and second-order derivative of $\pi_{b}$ with respect to $\hat{p}$, it follows that $d \pi_{b} / d \hat{p}=p \bar{F}\left(\hat{x}^{*}\right) \cdot d \hat{x}^{*} / d \hat{p}-\hat{p} \cdot d \hat{x}^{*} / d \hat{p}-\hat{x}^{*}$ and $d^{2} \pi_{b} / d \hat{p}^{2}$ $=\left[p \bar{F}\left(\hat{x}^{*}\right)-\hat{p}\right] \cdot d^{2} \hat{x}^{*} / d \hat{p}^{2}-p f\left(\hat{x}^{*}\right) \cdot\left(d \hat{x}^{*} / d \hat{p}\right)^{2}-2 d \hat{x}^{*} / d \hat{p} . \mathrm{It}$ 's easy to deduce that $d^{2} \pi_{b} / d \hat{p}^{2}<0$ since $d \hat{x}\left(q^{B^{*}}\right) / d \hat{p}>0$ and $d^{2} \hat{x}\left(q^{B^{*}}\right) / d \hat{p}^{2}<0$. Hence, let $d \pi_{b} / d \hat{p}^{*}=0$, we have $d \hat{x}^{*} / d \hat{p}^{*}=\left[w \bar{F}\left(q^{B^{*}}\right)-L f\left(q^{B^{*}}\right)\right] /\left[\hat{p}^{2} f\left(q^{B^{*}}\right)-w^{2} f\left(\hat{x}\left(q^{B^{*}}\right)\right)\right]$. Furthermore, since $\hat{p}^{*}=p / 1+R_{r}^{*}$ the optimal interest rate can be obtained as $R_{r}^{*}=w^{2}\left(p-\operatorname{Lg}\left(\hat{x}\left(q^{B^{*}}\right)\right)\right)-p^{2}\left(w \bar{F}\left(q^{B^{*}}\right)-\operatorname{Lf}\left({\left.q^{B^{*}}\right)}^{2}\right) / w^{2} \operatorname{Lg}\left(\hat{x}\left(q^{B^{*}}\right)\right)\right.$.

From proposition 2 it is obvious that the bank's optimal financing rate for the capital-constrained retailer is dependent on the borrower's financial conditions (i.e., initial capital, credit line, and so on), as well as his operational parameters (i.e., retailing price, order quantity, wholesale price). It also shows that there exists an inextricable relationship between operational decisions and financing decisions.

\section{Manufacturer's Optimal Decision}

In this paper, we assume that the manufacturer has unlimited production capacities and produces at minimum as much goods to fulfill the retailer's demand. As the leader of the above Stackelberg game, the manufacturer's decision is to choose an optimal wholesale price for maximizing the expected profit under the given financing mode, which can be expressed as Eq. (3).

$$
\max _{w} \pi_{m}(w, q)=(w-c) q^{B^{*}}
$$

According to the analysis in section 3.1, the manufacturer will respond to the retailer by setting an optimal wholesale price based on whether the retailer is capital constrained.

Proposition 3. If the demand distribution is IFR, the manufacturer's optimal wholesale price $w^{B^{*}}$ for the capital-constrained retailer with supply chain financing is determined as $w^{B^{*}}=p c /\left[p\left(1-G\left(q^{B^{*}}\right)\right)+c q^{B^{*}}\left(1+R_{r}^{*}\right) g\left(\hat{x}\left(q^{B^{*}}\right)\right)\right]$.

Proof. From Eq. (3), taking the first-order and second-order derivative of $\pi_{m}$ with respect to $w$, we have $d \pi_{m} / d w=(w-c) \cdot d q^{B^{*}} / d w+q^{B^{*}} \quad$ and $d^{2} \pi_{m} / d w^{2}=2 \cdot d q^{B^{*}} / d w+(w-c) \cdot d^{2} q^{B^{*}} / d w^{2}$. From the first-order condition of $d \pi_{m} / d w^{B^{*}}=0$, the optimal wholesale price can be obtained as $w^{B^{*}}=c\left[\hat{p}-w^{B^{*}} q^{B^{*}} g\left(\hat{x}\left(q^{B^{*}}\right)\right)\right] / \hat{p}\left(1-G\left(q^{B^{*}}\right)\right) \quad$, i.e., $w^{B^{*}}=p c / p\left(1-G\left(q^{B^{*}}\right)\right)+c q^{B^{*}}\left(1+R_{r}^{*}\right) g\left(\hat{x}\left(q^{B^{*}}\right)\right)$.

It's obvious from Proposition 3 that $w^{B^{*}}>c$ since $G\left(q^{B^{*}}\right) \leq 1$. Therefore, the optimal wholesale prices are effective to guarantee the manufacturer's positive profit.

Therefore, mathematically, the above three Propositions can be combined together to get the equilibrium solutions of the proposed Stackelberg game for supply chain financing. Through calculations and analysis, we found that even the demand distributions are known the closed-form analytical expression of these equilibriums are difficult to solve. However, the specific probability density and distribution 
function of the demand for each product in practical operations are usually unavailable.

\section{Robust DECISIONS OF SCF}

Next, we assume that through the analysis of historical data and forecasts, we can only get the region of demand interval at best, i.e. $x \in[A, B]$ and $B \geq A$, not knowing the specific demand distribution. So, how to design the robust strategies which is best in any worst demand case for each node company in supply chain under demand uncertainties are of great practical significance. According to the definition of robustness in [10], we analyze the deviation robustness of supply chain financing decisions.

\section{A. Retailer's Robust Decision}

In this section we would provide a solution of robust order quantity $q^{R}$ which minimizes the maximum profit loss due to demand uncertainty over all choices of order quantities. This is a minimax regret approach in which the regret is captured by the difference $\pi(x, x)-\pi\left(q^{R}, x\right)$, namely, the profit that could be realized if there was no demand uncertainties minus the profit made for the order quantity $q^{R}$. Note, in this case the retailer would set its order $q^{R}=x$. The maximum regret refers to the maximum cost of information collecting in order to obtain specific demand distribution knowledge.

Here, the deviation robust problems for the unconstrained retailer can be expressed as Eq. (4).

$$
\begin{gathered}
\Theta_{r}^{N R}\left(q^{N R}\right)=\min \Theta\left(q^{N R}\right)=\min _{q^{N R} \geq 0} \max _{x \in[A, B]}\left(\pi_{r}(x, x)-\pi_{r}\left(q^{N R}, x\right)\right) \\
=\min _{q^{N R} \geq 0} \max _{x \in[A, B]}\left\{(p-w) x-p E\left[\min \left(x, q^{N R}\right)\right]+w q^{N R}\right\}
\end{gathered}
$$

Similarly, the deviation robust problem for the capital-constrained retailer is formulated as Eq. (5).

$$
\begin{aligned}
& \Theta_{r}^{B R}\left(q^{B R}\right)=\min \Theta\left(q^{B R}\right)=\min _{q^{B R} \geq 0} \max _{x \in[A, B]}\left(\pi_{r}^{B}(x, x)-\pi_{r}^{B}\left(q^{B R}, x\right)\right) \\
& =\min _{A \leq q^{B R} \leq B}\left(\max _{A \leq q^{B R} \leq B}\left[p-w\left(1+R_{r}\right)\right] x-p \cdot \min \left(x, q^{B R}\right)+w q^{B R}\left(1+R_{r}\right)\right)
\end{aligned}
$$

Proposition 4. For, the retailer's robust order of the unconstrained supply chain is $q^{N R}=B-\beta^{N}(B-A)$, and the resulting deviation robust profit is $\Theta^{N R}\left(q^{N R}\right)=w\left(1-\beta^{N}\right)(B-A) \quad$. In addition, the capital-constrained retailer's robust order with the interest rate $R_{r}$ is $q^{B R}=B-\beta^{N}\left(1+R_{r}\right)(B-A)$, and his deviation robust profit with supply chain financing is $\Theta^{B R}\left(q^{B R}\right)=p(B-A) \beta^{N}\left(1+R_{r}\right)\left[1-\beta^{N}\left(1+R_{r}\right)\right]$.

Proof. Eq. (4) can be rewritten as follows,

$$
\begin{aligned}
& \Theta^{N R}\left(q^{N R}\right)= \begin{cases}\min _{A \leq q^{N R} \leq B}\left(\max _{A \leq q^{N R} \leq B}(p-w)\left(x-q^{N R}\right)\right) & x \geq q^{N R} \\
\min _{A \leq q^{N R} \leq B}\left(\max _{A \leq q^{N R} \leq B}(w-v)\left(q^{N R}-x\right)\right) & x<q^{N R}\end{cases} \\
& =\min _{A \leq q^{N R} \leq B}\left[\max \left((w-v)\left(q^{N R}-A\right),(p-w)\left(B-q^{N R}\right)\right)\right] .
\end{aligned}
$$

Hence, the robust deviation order for the unconstrained retailer is $\quad q^{N R}=A w+B(p-w) / p=B-\beta^{N}(B-A) \quad$. Substituting $q^{N R}$ into $\Theta^{N R}\left(q^{N R}\right)$, we have $\Theta^{N R}\left(q^{N R}\right)=w\left(1-\beta^{N}\right)(B-A)$.

Similarly, Eq. (5) can be rewritten as follows,

$$
\begin{aligned}
& \Theta^{B R}\left(q^{B R}\right)=\left\{\begin{array}{lc}
\min _{A \leq q^{B R} \leq B}\left(\max _{A \leq q^{B R} \leq B}(p-w)\left(x-q^{B R}\right)\right) & x \geq q^{B R} \\
\min _{A \leq q^{B R} \leq B}\left(\max _{A \leq q^{B R} \leq B}(w-v)\left(q^{B R}-x\right)\right) & x<q^{B R}
\end{array}\right. \\
& =\min _{A \leq q^{B R} \leq B}\left(\max \left(w\left(1+R_{r}\right)\left(q^{B R}-A\right),\left(p-w\left(1+R_{r}\right)\right)\left(B-q^{B R}\right)\right)\right)
\end{aligned}
$$

Hence, the robust deviation order for the retailer is $q^{B R}=\left[A w\left(1+R_{r}\right)+B\left(p-w\left(1+R_{r}\right)\right)\right] / p=B-\beta^{N}\left(1+R_{r}\right)(B-A)$. Substituting $q^{B R}$ into $\Theta^{B R}\left(q^{B R}\right)$, it follows that $\Theta^{B R}\left(q^{B R}\right)=p(B-A) \beta^{N}\left(1+R_{r}\right)\left[1-\beta^{N}\left(1+R_{r}\right)\right]$.

\section{B. Bank's Robust Decision}

In the supply chain financing system with a capital-constrained retailer, the commercial bank, as one of the key participants, he may differentiate the interest rate to realize profit maximization according to robust order quantities. Similar to the above analysis, we formulate the bank's robust decision problem as Eq. (6).

$\max \pi_{b}^{B R}\left(R^{B R} ; q^{B R}, x\right)=\min \left\{L\left(q^{B R}\right)\left(1+R^{B R}\right), p \min \left(q^{B R}, x\right)\right\}-L\left(q^{B R}\right)$

Proposition 5. For $x \in[A, B]$, the robust interest rate determined by the bank under given initial capital and the wholesale price is expressed as $R_{r}^{B R}=\left[p\left(w B-K_{r}\right)-w^{2}(B-A)\right] / 2 w^{2}(B-A), \quad$ and the bank's robust profit is $\pi_{b}^{B R}\left(q^{B R}, R_{r}^{B R}\right)=$ $p\left(w B-K_{r}\right)^{2} / 4 w^{2}(B-A)-\left(w B-K_{r}\right) / 2+w^{2}(B-A) / 4 p$.

Proof. From Eq. (6), taking the first-order and second-order derivative of $\pi_{b}^{B R}$ with respect to $R_{r}^{B R}$, we have $\quad d \pi_{b}^{B R} / d R_{r}^{B R}=w q_{r}^{B R}-K_{r}-R_{r}^{B R}(B-A) w \beta^{N} \quad$ and $d^{2} \pi_{b}^{B R} / d R_{r}^{B R 2}=-\beta^{N}(1+w)(B-A)<0 \quad$. Hence, let $d \pi_{b}^{B R} / d R_{r}^{B R^{*}}=0$, we have $R_{r}^{B R}=p\left(w q^{B R}-K_{r}\right) / w^{2}(B-A)$ $=p\left(w B-K_{r}\right)-w^{2}(B-A) / 2 w^{2}(B-A)$. Substituting $R_{r}^{B R}$ into $\pi_{b}^{B R}$, it follows that $\pi_{b}^{B R}\left(q^{B R}, R_{r}^{B R}\right)=$ $p\left(w B-K_{r}\right)^{2} / 4 w^{2}(B-A)-\left(w B-K_{r}\right) / 2+w^{2}(B-A) / 4 p$.

\section{Manufacturer's Robust Decision}

Considering the retailer's robust order quantity, the manufacturer makes conservative pricing decisions to seek maximum expected profit.

In the unconstrained supply chain, the manufacturer's decision problem with the retailer's robust decision is:

$$
\max _{c \leq w^{N R} \leq p} \pi_{m}^{N R}\left(w^{N R}, q^{N R}\right)=\left(w^{N R}-c\right) q^{N R}
$$

Then, in the capital-constrained supply chain, his decision problem is formulated as Eq. (8). 


$$
\max _{c \leq w^{B R} \leq p} \pi_{m}^{B R}\left(w^{B R}, q^{B R}\right)=\left(w^{B R}-c\right) q^{B R}
$$

Proposition 6. For $x \in[A, B]$, the manufacturer's robust wholesale price $w^{N R}$ for the unconstrained retailer and $w^{B R}$ for the capital-constrained retailer with supply chain financing are determined as $w^{N R}=c / 2+B p / 2(B-A)$ and $w^{B R}=c / 2+B p /\left[2(B-A)\left(1+R_{r}^{B R}\right)\right]$.

Proof. In the unconstrained supply chain system, taking the first-order and second-order derivative of $\pi_{m}^{N R}$ with respect to $w^{N R}$, it follows that $d \pi_{m}^{N R} / d w^{N R}=\left(w^{N R}-c\right) \cdot d q^{N R} / d w^{N R}+q^{N R} \quad$ and $d^{2} \pi_{m}^{N R} / d w^{N R 2}=-2(B-A)\left(1+R_{f}\right) /(p-v)<0$. Hence, from the first-order condition of $d \pi_{m} / d w^{N R}=0$, we have $w^{N R}=\left[c(B-A)\left(1+R_{f}\right)-A v+B p\right] / 2(B-A)\left(1+R_{f}\right)$.

Similarly, in the capital-constrained supply chain, from Eq. (8), taking the first-order and second-order derivative of $\pi_{m}^{B R} \quad$ with respect to $w^{B R}$, we have $d \pi_{m}^{B R} / d w^{B R}=\left(w^{B R}-c\right) \cdot d q^{B R} / d w^{B R}+q^{B R} \quad$ and $d^{2} \pi_{m}^{B R} / d w^{B R 2}=-2(B-A)\left(1+R_{r}\right) / p<0$. Hence, from $d \pi_{m}^{B R} / d w^{B R}=0 \quad, \quad$ we have $w^{B R}=\left[c(B-A)\left(1+R_{r}^{B R^{*}}\right)-A v+B p\right] / 2(B-A)\left(1+R_{r}^{B R^{*}}\right)$.

It's obvious from Proposition 6 that $\partial w^{B R} / \partial R_{r}^{B R}<0$, which implies that robust wholesale price decreases in the robust interest rate. Moreover, it's straightforward that $w^{B R} \leq w^{N R}$ since $R_{r}^{B R} \geq R_{f}$, which implies that the robust wholesale price of supply chain financing is not more than that of the unconstrained supply chain. In other words, considering the retailer's capital constraints the manufacturer would offer a lower wholesale price to ease the cash gap and financing costs and the resulting bankruptcy risk.

\section{NUMERICAL ANALYSIS}

To further explore the impact of demand uncertainty on the decisions made in SCF, we conduct a computational study, showing that generally, as we would expect, robust decisions with unknown demands are conservative compared with optimal decisions under given demand distribution. Specifically, using numerical examples we illustrate the above comparative statics to discuss the differences between robust decisions and optimal decisions under different demand uncertainties (as captured by the region of demand intervals).

For parameter setting, we assume that $p=1, c=0.3, K_{r}=1.5, R_{f}=0$, the interval of demand with unknown distribution is $x \sim[A, B]$ and the known mean is $\mu=10$. According to the above analytical discussion, we make the following enriched observations through some numerical examples based on different demand intervals, i.e., $B-A$. In order to explore the impact of the demand distribution on the optimal decisions for each decision-maker in SCF, we compare the scenarios with uniform, normal and gamma distributions and the robust deviation without known distribution, depicted in Fig. 1-Fig. 6.

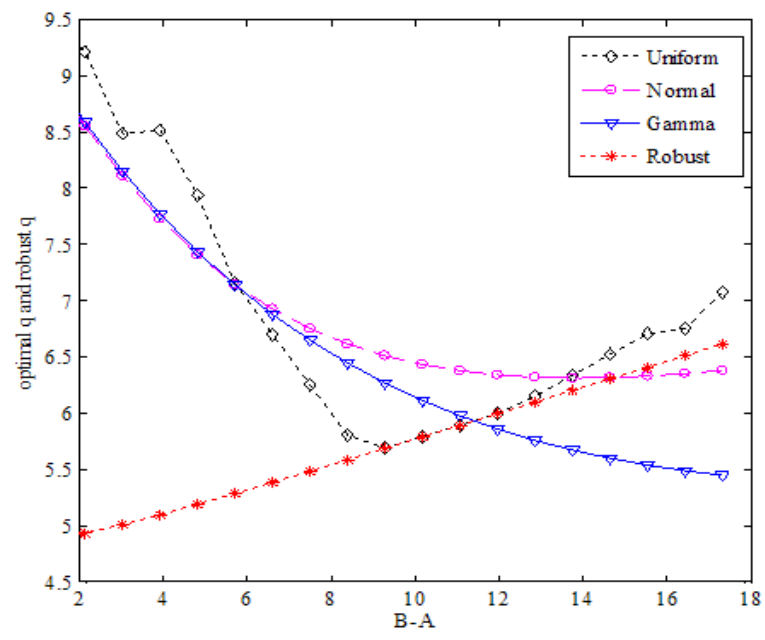

Fig. 1. Optimal and robust orders with different demand intervals.

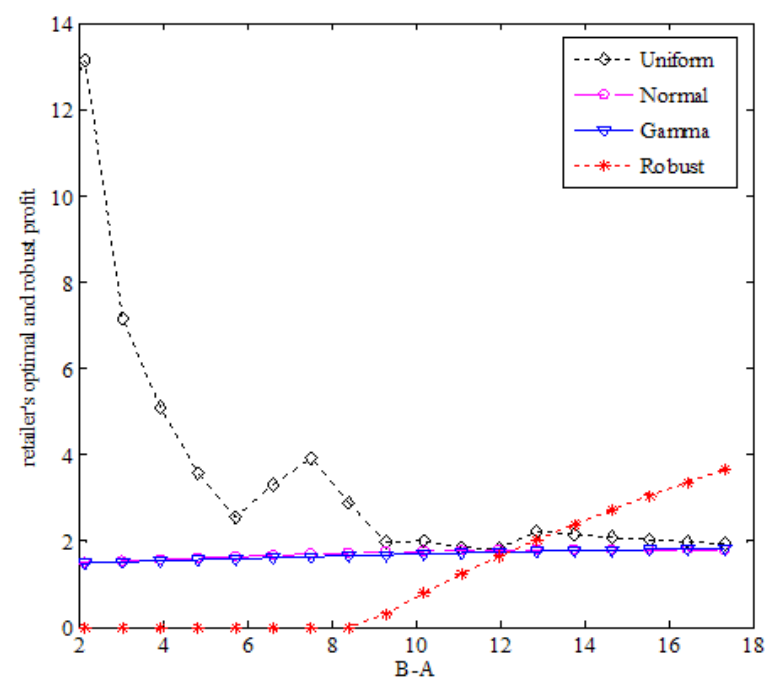

Fig. 2. Retailer's optimal and robust profits with different demand intervals

Fig. 1 demonstrates that the retailer's robust order increases in the demand interval, and the differences between the robust order and the optimal orders with known distribution becomes small. It implies that as the demand interval increases, the demand uncertainties become less influential. Note that when the interval is smaller the robust order is rather conservative compared with optimal orders. It also results in less profit, as depicted in Fig.2. It's obvious in Fig. 2 that the robust profit is less than the optimal profits if the demand uncertainty is small. As the demand interval increases, the distribution information greatly affects the robust profit, but slightly on the optimal profits.

Fig. 3 and Fig. 4 depict the manufacturer's pricing decisions and the resulting profits. The robust wholesale price and profit decrease in the demand uncertainty. The conservative manufacturer charges a higher robust price than optimal prices to the capital-constrained retailer for risk aversion if the demand interval is small. Generally, regardless of the demand uncertainty, the robust profit maintains less than the corresponding profits under optimal pricing decision. 
Furthermore, we compute the bank's optimal and robust interest rates and the resulting profits, depicted in Fig. 5 and Fig. 6. According to the retailer's ordering decision and manufacturer's pricing decision, the bank's robust interest rate firstly increases and then decreases with the increasing of the demand uncertainty. Moreover, similar phenomenon can be observed in Fig. 6 when compared with the bank's optimal expected profits under different demand distribution. There is no significant difference in optimal profits between these three distributions, especially with higher demand uncertainty. However, the robust profit is obviously less than the optimal profits with less demand intervals.

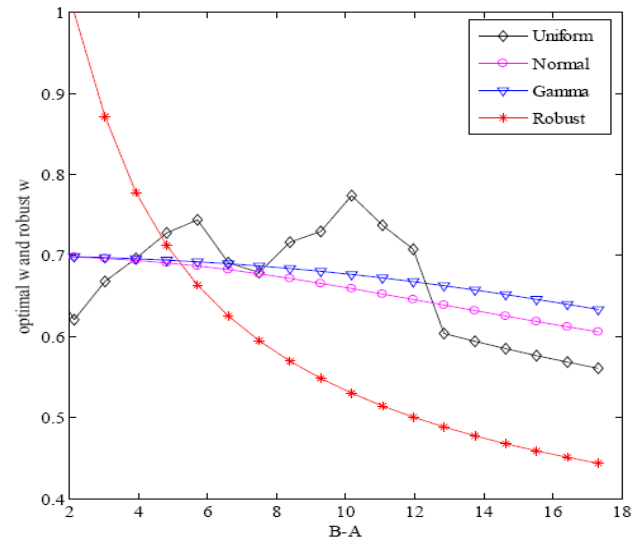

Fig. 3. Optimal and robust wholesale prices with different demand intervals.

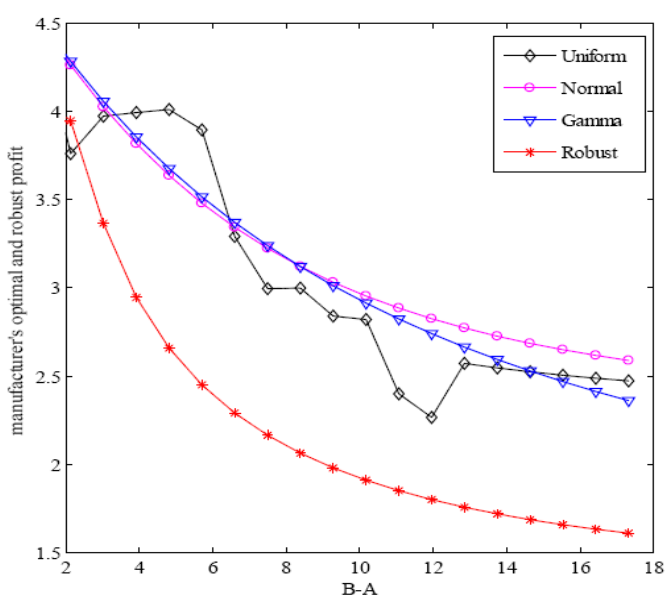

Fig. 4. Manufacturer's optimal and robust profits with different demand intervals.

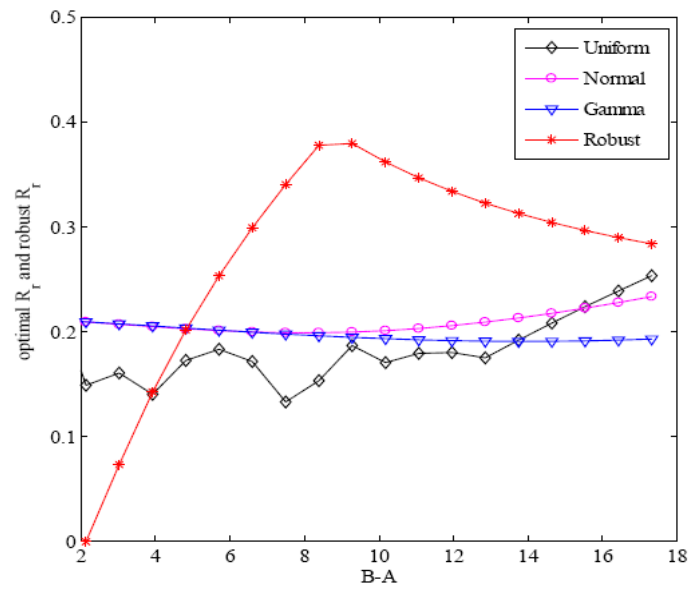

Fig. 5. Optimal and robust interest rates with different demand intervals.

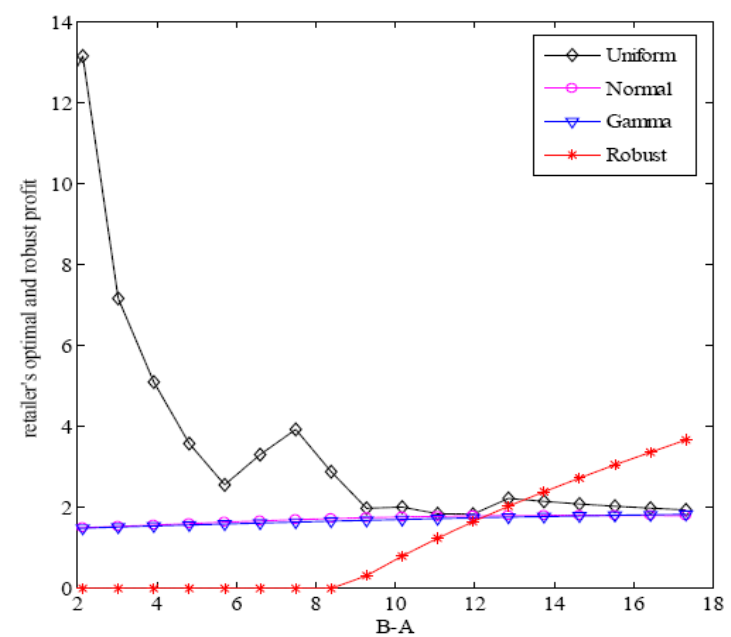

Fig. 6. Bank's optimal and robust profits with different demand intervals.

\section{CONCLUSION}

In this paper, we tried to take robust decisions into considerations in supply chain financing games, composed of

a capital-constrained retailer, a manufacturer and a commercial bank. In particular, we focused on the different equilibriums under different demand uncertainties, i.e., the optimal decisions with given demand distribution and the robust deviations only knowing the region of demand intervals.

Through our analysis, we establish the following major results. First, the optimal decision under the objective of profit maximization need to know the specific demand distribution and the closed-form analytical expressions of these equilibriums are difficult to solve. Second, the robust decision based on minimize-maximum-regret provides a conservative strategy for risk aversion. Moreover, it is clear from the analysis that the region of demand intervals has great impact on robust deviations, rather than the optimal decisions. Specifically, the robust interest rate and wholesale price are higher than the counterparts of optimal decisions under given demand distributions.

However, our robust analysis of SCF is a tentative investigation. In this paper, we ignore the analysis of risk preference, which is worthy to be studied further.

\section{REFERENCES}

[1] H. C. Pfohl and M. Gomm, "Supply chain finance: optimizing financial flows in supply chains," Logistics Research, vol. 1, no. 3-4, pp.149-161, 2010.

[2] M. Dada and Q. Hu, "Financing newsvendor inventory," Oper. Res. Lett., vol. 36, no. 5, pp.569-573,2008

[3] X. Chen and A. Wang, "Trade credit contract with limited liability in the supply chain with budget constraints," Ann. Oper. Res., vol. 196, no. 1, pp. 153-165, 2012.

[4] B. Jing, X. Chen, and G. Cai, "Equilibrium financing in a distribution channel with capital constraint," Prod. Oper. Manage, vol. 21, no. 6 , pp.1090-1101, 2012.

[5] G. Zhang, "The multi-product newsboy problem with supplier quantity discounts and a budget constraint," Eur. J. Oper. Res., vol. 206, no. 2, pp. $350-360,2010$.

[6] C. H. Lee and B. D. Rhee, "Coordination contracts in the presence of positive inventory financing costs," Int. J. Prod. Econ., vol. 124, no. 2, pp. 331-339, 2010.

[7] N. R. S. Raghavan and V. K. Mishra, "Short-term financing in a cash-constrained supply chain," Int. J. Prod. Econ., vol. 134, no. 2, pp. zz407-412, 2011. 
[8] X. D. Xu and J. R. Birge. Joint Production and Financing Decisions: Modeling and Analysis. [Online]. Available: http://ssrn.com/absract $=652562$.

[9] P. Kouvelis and W. Zhao, "Financing the newsvendor: Supplier vs.bank, optimal rates, and alternative schemes," Working Paper, Olin Business School, Washington University, St. Louis, MO. 2008.

[10] G. Vairaktarakis, "Robust multi-item newsboy models with a budget constraint," Int. J. Prod. Econ., vol.66, no. 2, pp. 213-226, 2000.

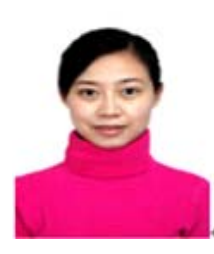

Nina Yan was born in China in 1980. She received the $\mathrm{PhD}$ in management science from Northeastern University, Shenyang, China in 2007, and engaged in the Postdoctoral researches in Renmin University and Shougang Iron\& Steel Research Academy, Beijing, China, from 2007 to 2009.

She is an associate professor of Business School in Central University of Finance and Economics. Her research interests are in supply chain management, operations management and e-commerce. She has published over 30 papers in Chinese and International Journals, such as International Journal of Production Research, OR Spectrum, Asia-Pacific Journal of Operational Research, OR Spectrum and so on. 